\title{
AcouStick: A Tracked A-Mode Ultrasonography System for Registration in Image-Guided Surgery
}

Calvin R. Maurer, Jr. ${ }^{1,2}$, Ronald P. Gaston ${ }^{2}$, Derek L. G. Hill ${ }^{2}$, Michael J. Gleeson ${ }^{3}$, M. Graeme Taylor ${ }^{2}$, Michael R. Fenlon ${ }^{4}$, Philip J. Edwards ${ }^{2}$, and David J. Hawkes ${ }^{2}$

1 Departments of Neurological Surgery and Biomedical Engineering University of Rochester, 601 Elmwood Avenue, Box 670, Rochester, NY 14642, USA

Calvin_Maurer@URMC. Rochester . edu

2 Division of Radiological Sciences and Medical Engineering King's College London

5th Floor Thomas Guy House, Guy's Hospital, London SE1 9RT, UK

David.Hawkes@KCL . ac .uk

3 Department of Surgery, King's College London, Guy's Hospital, London, UK

${ }^{4}$ Department of Prosthetic Dentistry

King's College London, Guy's Hospital, London, UK

\begin{abstract}
In this paper, we describe a system for noninvasively determining bone surface points using an optically tracked A-mode ultrasound transducer. We develop and validate a calibration method; acquire cranial surface points for a skull phantom, three volunteers, and one patient; and register these points to surfaces extracted from CT images of the phantom and patient. Our results suggest that the bone surface point localization error of this system is less than $0.5 \mathrm{~mm}$. The target registration error (TRE) of the cranial surface-based registration for the skull phantom was computed by using as a reference gold standard the point-based registration obtained with eight bone-implanted markers. The mean TRE for a 150-surface-point registration is $1.0 \mathrm{~mm}$, and ranges between 1.0 and $1.7 \mathrm{~mm}$ for six 25-surface-point registrations. Our preliminary results suggest that accurate, noninvasive, image-tophysical registration of head images may be possible using an A-mode ultrasound-based system.
\end{abstract}

\section{Introduction}

Registration of preoperative images with the physical space occupied by the patient during surgery is a fundamental step in image-guided surgery. Imageto-physical space registration is commonly performed using stereotactic frame systems, points, and surfaces. Point-based registration can be accomplished using external anatomic landmarks (e.g., internal and external canthi, nasion, tragus), skin-affixed markers, and bone-implanted markers. Surface-based registration is generally accomplished using the air-skin interface. Registration using the 
bone surface could remove errors due to deformation of the skin surface between scanning and the operation.

In this paper, we describe a system for noninvasively determining cranial surface points using an optically tracked A-mode ultrasound transducer. These points can be used to perform surface-based image-to-physical registration of three-dimensional (3-D) head images. We develop and validate a calibration method; acquire cranial surface points for a skull phantom, three volunteers, and one patient; and register these points to surfaces extracted from CT images of the phantom and patient.

\section{Methods}

\subsection{System Overview}

In pulse echo ultrasound, a short pulse of energy is transmitted into the body. Echoes in the received signal represent sound reflected at interfaces between regions of different acoustic impedance. The intensity reflection coefficient, which is the ratio of the pressure reflected to the pressure incident, is less than 0.1 for most soft tissue interfaces, and is approximately $0.6-0.7$ for bone-tissue interfaces [8]. Thus echoes corresponding to bone-tissue interfaces have high signal amplitude and are easily identified. The distance from the transducer to the interface corresponding to an echo is easily calculated as $d=u t / 2$, where $d$ is the distance, $u$ is the speed of sound, and $t$ is the time interval between the initial sound pulse and the received echo. Thus our aim is to track the position of an ultrasound transducer and calculate the position of a bone surface point as the position of the transducer face plus the distance to the bone-tissue interface along the ultrasound beam axis.

We use a heavily damped, spherically focused, $10 \mathrm{MHz}$ A-mode ultrasound immersion transducer (part number V312, Panametrics, Waltham, MA). The nominal element size is $6 \mathrm{~mm}$ and the focal length in water at $22{ }^{\circ} \mathrm{C}$ is approximately $19 \mathrm{~mm}$. The $-6 \mathrm{~dB}$ beam width at the focal point is $0.4 \mathrm{~mm}$. The $-6 \mathrm{~dB}$ depth of field is $10 \mathrm{~mm}$.

The transducer is driven with an ultrasonic pulser/receiver (model 5072PR, Panametrics). The damping is set to $50 \mathrm{ohm}$. The amplifier gain is varied between 0 and $20 \mathrm{~dB}$ as necessary to maximize reflected signal amplitude without clipping. The pulse repetition frequency is set to $100 \mathrm{~Hz}$. The pulser/receiver output is connected to a 12-bit, $80 \mathrm{MHz}$ analog-to-digital (A/D) data acquisition board (CompuScope 8012/PCI, Gage, Montreal, Canada) placed in a PC running Windows NT. The A/D board is operated in single-channel capture mode with a sampling rate of $80 \mathrm{MHz}$. The input voltage range is $\pm 1 \mathrm{~V}$ and the input signal is DC coupled. Signal acquisition is controlled by a program written in Microsoft $\mathrm{C}++$ using the win32 API. The A/D board is programmed to collect data continuously and store a fixed number of samples in a circular buffer after a trigger event is detected. The trigger level is set at $500 \mathrm{mV}$ with a positive trigger slope. This provides a reliable and reproducible trigger on the 


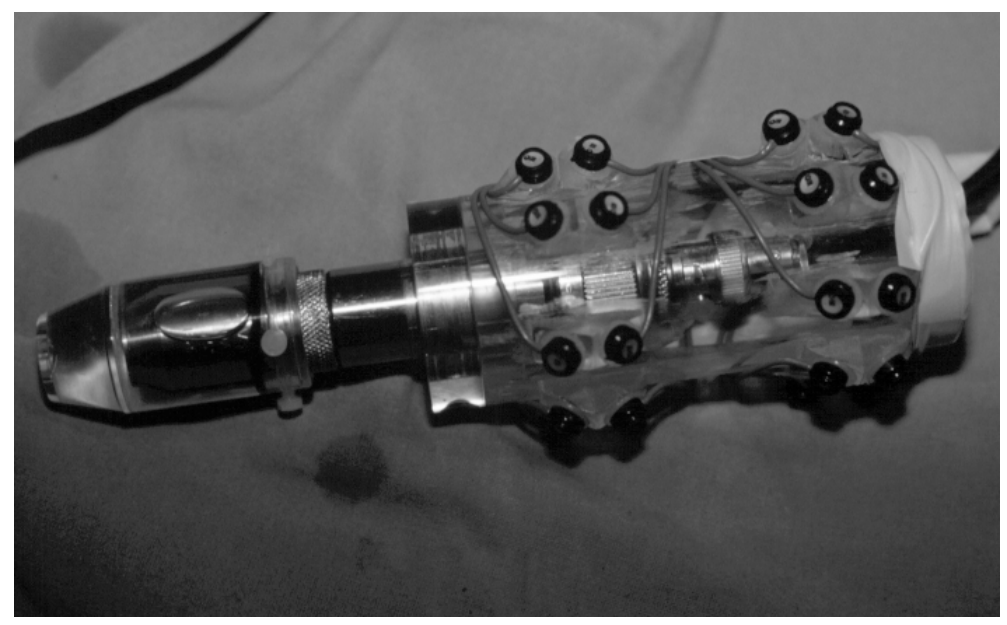

Fig. 1. Photograph showing the A-mode ultrasound transducer, the array of attached IREDs, and the offset.

initial signal of the pulser/receiver pulse. We store 8192 samples $(102 \mu \mathrm{s})$ per pulse. This corresponds to $79 \mathrm{~mm}$ if the speed of sound is $1540 \mathrm{~m} / \mathrm{s}$.

The ultrasound transducer is optically tracked using an Optotrak 3020 system (Northern Digital, Inc., Waterloo, Ontario, Canada). Twenty infrared lightemitting diodes (IREDs) are mounted on a plastic cylindrical housing attached to a UHF-to-UHF connector (immersion search tube, part number F112, Panametrics) that is screwed into the back of the transducer.

A plastic (polyetherimide, Ultem, General Electric) offset is attached to the transducer. Polyetherimide was used because it can be autoclaved. The tip of the offset is adjustable. It is normally set at about $15 \mathrm{~mm}$ from the transducer face so that the transducer focal point is approximately at the cranial surface. The offset is filled with water during data collection and has a hole in the tip for water coupling at the skin surface. The ultrasound transducer, the array of IREDs, and the offset are shown in Fig. 1.

Echo locations in the received ultrasound signal are determined using the Hilbert transform. The envelope of the received signal is calculated as the magnitude of the analytic signal. The analytic signal of $x(t)$ is the complex signal that has $x(t)$ as its real part and the Hilbert transform of $x(t)$ as its imaginary part. The Hilbert transform is related to the actual data by a $\pi / 2$ phase shift. We compute the Hilbert transform in the time domain using a finite-impulse response (moving average) filter of order 60. The filter coefficients were calculated with the Parks-McClellan algorithm using the Matlab (The Mathworks, Inc., Natick, MA) signal processing toolbox function remez. We define the echo locations as the maxima of the echo peaks in the resulting signal envelope. A 


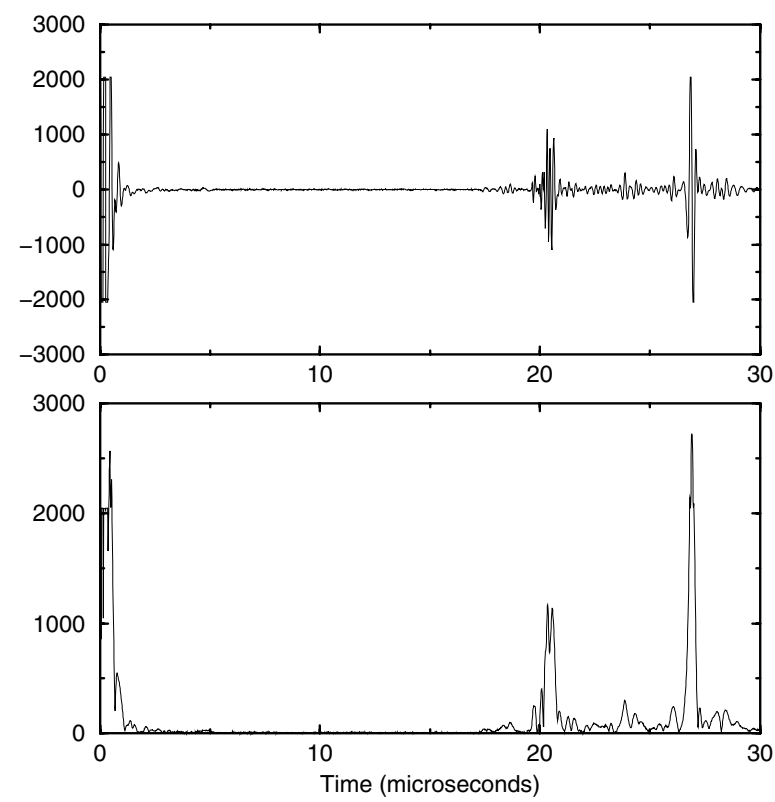

Fig. 2. A sample ultrasound signal from a volunteer (top) and the magnitude of the analytic signal (bottom). The initial sound pulse is at $t=0$. The first received echo at $t \approx 20 \mu \mathrm{s}$ corresponds to the water-skin interface. The second echo at $t \approx 27 \mu s$ corresponds to the tissue-bone interface. This data is typical of the signals we obtain.

sample ultrasound signal from a volunteer and the magnitude of the analytic signal are shown in Fig. 2.

\subsection{Calibration}

The AcouStick is essentially a tracked probe with a variable length tip. Cranial surface positions $\mathbf{p}$ are calculated as $\mathbf{p}=\mathbf{p}_{0}+d \mathbf{n}_{p}$, where $\mathbf{p}_{0}$ is the position of the transducer, i.e., the 3 -D point corresponding to time zero in Fig. $2, d=u t / 2$ is the distance from the transducer to the bone-tissue interface, $u$ is the speed of sound in tissue, $t$ is the time interval between the initial sound pulse and the bone-tissue echo, and $\mathbf{n}_{p}$ is the unit vector along the direction of the ultrasound beam. Calibration for this system is the process of determining $\mathbf{p}_{0}$ and $\mathbf{n}_{p}$.

Our method is a variation of the invariant point method commonly used for calibrating ball-tipped probes. We mount a steel ball on a post attached to a heavy base and place it in a tracked water bath. The diameter of the ball we use is $7 \mathrm{~mm}$. The ultrasound probe is placed in the water and moved until a strong echo reflected from the ball is observed in the ultrasound signal. We calculate the position of the center of the steel ball using $d=u t / 2+r$, where $u$ is the speed of sound in water at the temperature of the bath and $r$ is the radius of the 
sphere. There are three coordinate systems in this process: the probe coordinate system $P$, the world coordinate system $W$ defined by the optical position sensor, and the reference coordinate system $R$ defined by an array of IREDs that we place on the water bath. The calculated position of the steel ball in the reference coordinate system, ${ }^{R} \mathbf{p}_{i}$, is

$$
{ }^{R} \mathbf{p}_{i}={ }^{R} T_{P i}{ }^{P} \mathbf{p}_{i}={ }^{R} T_{W i}{ }^{W} T_{P i}\left(\mathbf{p}_{0}+d_{i} \mathbf{n}_{p}\right),
$$

where ${ }^{R} T_{P i}$ is the rigid-body probe-to-reference transformation, ${ }^{P} \mathbf{p}_{i}$ is the position of the steel ball in the probe coordinate system, ${ }^{R} T_{W i}$ is the world-toreference transformation, and ${ }^{W} T_{P i}$ is the probe-to-world transformation. The transformations ${ }^{R} T_{W i}$ and ${ }^{W} T_{P i}$ are provided by the optical tracking system. The subscript $i$ denotes a single measurement. The position of the steel ball in the reference coordinate system, $\mathbf{w}_{\mathbf{0}}$, is fixed, and thus ${ }^{R} \mathbf{p}_{i} \approx \mathbf{w}_{\mathbf{0}}$. This relationship is not exact because of measurement error. Thus we iteratively search for the parameters $\mathbf{p}_{0}$ and $\mathbf{n}_{p}$ and the most invariant steel ball position $\mathbf{w}_{\mathbf{0}}$ in a least-squares sense, that is, we minimize $\chi_{\text {cal }}$ defined as

$$
\chi_{\text {cal }}^{2}=\sum_{i}\left\|{ }^{R} \mathbf{p}_{i}-\mathbf{w}_{\mathbf{0}}\right\|^{2} .
$$

We use a large number of points (approximately 24) collected at different orientations and distances. The probe is hand held and moved until a strong echo is detected. We perform calibration with the offset removed. This allows us to collect points over a wide range of lengths, from approximately $d=5$ to $60 \mathrm{~mm}$.

\subsection{Point Localization Error}

To estimate the AcouStick point localization error, we used the AcouStick system to measure the position of 255 -mm diameter steel balls arranged in a $5 \times 5$ grid on a acrylic plate in a water bath. The grid spacing is $10 \mathrm{~mm}$. We performed this measurement in two ways. In the first way, the probe is held with a hand and moved until a strong echo is detected. Each point measurement is obtained from a different orientation. We refer to this approach as the "free hand" method. In the second way, the probe is rigidly held in a clasp that is connected to a micrometer-controlled positioning stage. The probe is translated back and forth in two directions using the micrometer dials until the locally maximum echo amplitude is found as judged by visual examination of the constantly updated analytic signal. We do not use the micrometer readings in any quantitative way. Rather we use the micrometer dials merely to achieve fine translational movement. We refer to this approach as the "fixed orientation" method. Both types of measurements are made over the range of lengths for which the system is intended to be used, that is, from approximately $d=15$ to $25 \mathrm{~mm}$. We collected two sets of free hand measurements, and two sets of fixed orientation measurements. As the two types of measurements were performed on different occasions, a separate calibration was done for each. Both calibrations were obtained with 24 
points. The measured point sets were registered to the known grid positions. The AcouStick localization error was estimated as the residual error after registering these point sets times $\sqrt{N /(N-2)}$, where $N=25$ [10]. This assumes that the localization error is identical, independent, zero-mean, isotropic, and normally distributed.

\subsection{Skull Phantom Experiment}

We used the AcouStick system to collect cranial surface points on a plastic skull phantom covered with a layer of gelatin (see left and middle panels of Fig. 3). Eight bone-implanted markers similar to those described in [6] were used for validation. Plastic marker bases were screwed into the top of the skull. A CT image volume of the phantom was acquired using a Philips Tomoscan SR 7000 scanner. The image volume contains 145 axial slices with a slice thickness of $1.0 \mathrm{~mm}$. Just prior to image acquisition, imaging caps containing iodinated contrast were attached to the marker bases. After image acquisition, the phantom was lowered upside down into a latex swimming cap filled with a warm solution of gelatin (150 g/l) that was allowed to set overnight. The thickness of the gelatin varied over the cranial surface from 3 to $16 \mathrm{~mm}$, with a mean thickness of $9 \mathrm{~mm}$. The next day, ten IREDs were glued onto the front of the skull and were used to define the reference coordinate system. Marker bases were identified by manual palpation, small cuts were made in the swimming cap, and physical space caps with a hemispherical divot that corresponds to the centroid of the imaging caps were attached to the bases. Physical space positions of the markers were determined by placing an optically tracked ball-tipped probe in the divot of each cap. The AcouStick was calibrated and 150 cranial surface points widely distributed over the head were collected. The speed of sound in the gelatin was determined by measuring the time of flight through a plastic cylinder filled with the same gelatin used to cover the skull. The nominal length of the cylinder was $30 \mathrm{~mm}$. A more accurate length was determined with digital calipers. The speed of sound in water was similarly measured to validate this technique and was found to differ from published values by less than $0.3 \%$.

A triangle set representation of the cranial surface was automatically extracted from the CT image volume [4]. The ultrasonically determined cranial surface points were registered to the image-derived surface model using an independent implementation [5] of the iterative closest point registration algorithm [2]. We calculated the target registration error (TRE) of the surface-based registration by using as a reference gold standard the point-based registration obtained with the eight markers [7]. Specifically, we calculated TRE as the distance between a point in CT mapped from physical space by the reference transformation and its position mapped by the transformation being evaluated. We performed this calculation at the center of every CT voxel inside the head and averaged the resulting values. 

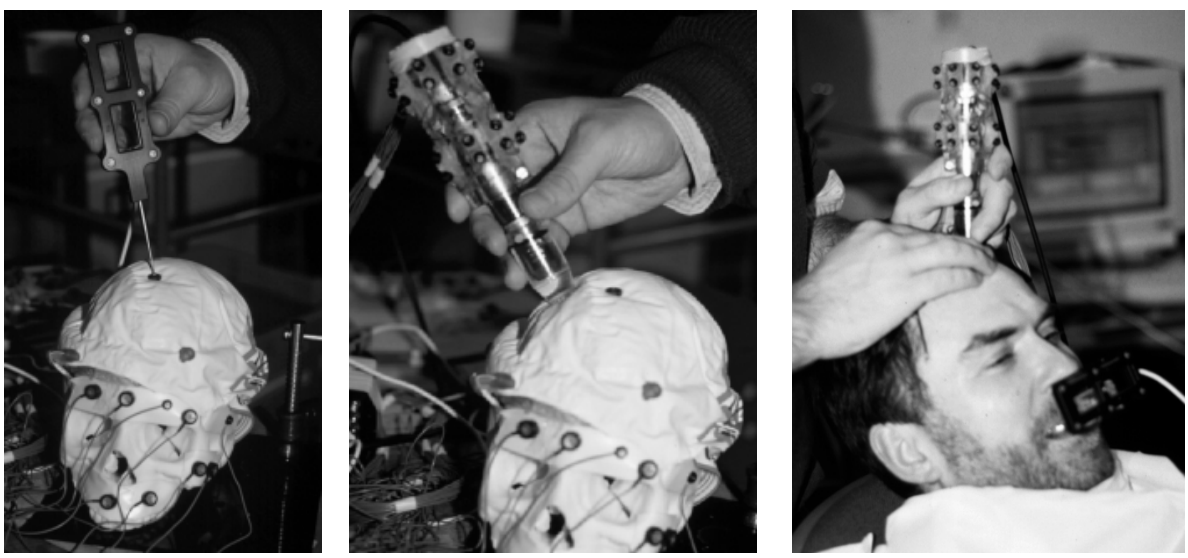

Fig. 3. Localization of markers (left) and collection of cranial surface points (middle) during the skull phantom experiment. Also, localization of skull surface points in one of the volunteer studies (right).

\subsection{Volunteer and Patient Data}

We used the AcouStick system to collect cranial surface points on three volunteers (see right panel of Fig. 3). Each volunteer had a locking acrylic dental stent (LADS). This custom built device attaches to the person's upper teeth. Six IREDs are attached to the LADS and are used to track head movement. The LADS assembly also contains ten fiducial markers similar to those in [6]. This allows the LADS device to also be used for image-to-physical registration if the device can be accurately repositioned. All three volunteers had an MR gradient echo image volume acquired while wearing the LADS. The ultrasonically determined cranial surface points were superimposed on the MR image volume according to the registration transformation provided by the LADS device and then visually examined.

We used the AcouStick system to collect cranial surface points on one patient that underwent surgery for removal of a petrous apex cyst. This patient had a CT image volume acquired as a normal part of his treatment. He was fitted with a LADS device that was used only for tracking of patient movement. The same methods used to extract a cranial surface model and perform surface-based registration for the skull phantom were used for the patient data.

\section{Results}

Calibrations performed with 24 points typically have a calibration residual error $\chi_{\text {cal }}$ of about $0.6-0.8 \mathrm{~mm}$. The calibration residual errors of the calibrations used for the free hand and fixed orientation validation measurements are 0.61 

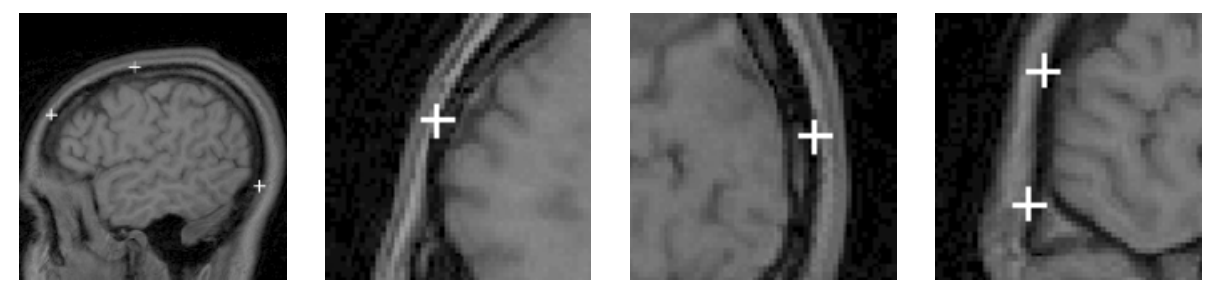

Fig. 4. Ultrasonically determined cranial surface points superimposed as white crosses on four regions from the MR image of a volunteer. The seven surface points shown are representative of those collected, and were transformed to image coordinates using the LADS-based registration.

and $0.75 \mathrm{~mm}$, respectively. The residual errors after registering the two free hand validation point sets to the known grid positions are 0.48 and $0.53 \mathrm{~mm}$. The residual errors for the fixed orientation validation point sets are 0.13 and $0.16 \mathrm{~mm}$.

The skull phantom cranial surface points determined using the AcouStick were registered to the triangle set representation of the cranial surface that was extracted from the CT image volume. Seven registrations were performed, one using all 150 AcouStick surface points, and six using randomly chosen independent subsets of 25 points each. The surface residual error, i.e., the rootmean-square difference between the surface points and the surface model after registration, is $0.20 \mathrm{~mm}$ for the 150 -point registration, and ranges between 0.10 and $0.18 \mathrm{~mm}$ for the 25-point registrations. The mean TRE over the head volume computed using the eight-marker point-based registration as a reference gold standard is $1.03 \mathrm{~mm}$ for the 150-point registration, and ranges between 1.04 and $1.73 \mathrm{~mm}$ for the 25-point registrations. The surface points superimposed on CT image slices using any of these surface-based transformations appear visually to lie on the cranial surface.

The cranial surface points collected from the three volunteers were superimposed on the volunteers' MR image volumes using the LADS-based transformation. For two of these data sets, visual inspection suggests that almost all of the surface points are within one or two voxels of the inner surface of the subcutaneous fat. The voxel dimensions of these images are approximately $1.0 \times 1.0$ $\times 1.5 \mathrm{~mm}$. Sample MR slices showing several of these surface points for one of the volunteers are shown in Fig. 4. For the third volunteer, there appears to be a systematic error in the position of the surface points. On one side of the head most of the points lie inside the bone by several millimeters, whereas on the other side of the head the points predominantly lie outside the bone by several millimeters. It is possible that this observation is caused by a systematic calibration or localization error. It is also quite plausible that it results from a LADS relocation error relative to its position during image acquisition.

The 30 cranial surface points collected from the patient using the AcouStick were registered to the triangle set representation of the cranial surface that 

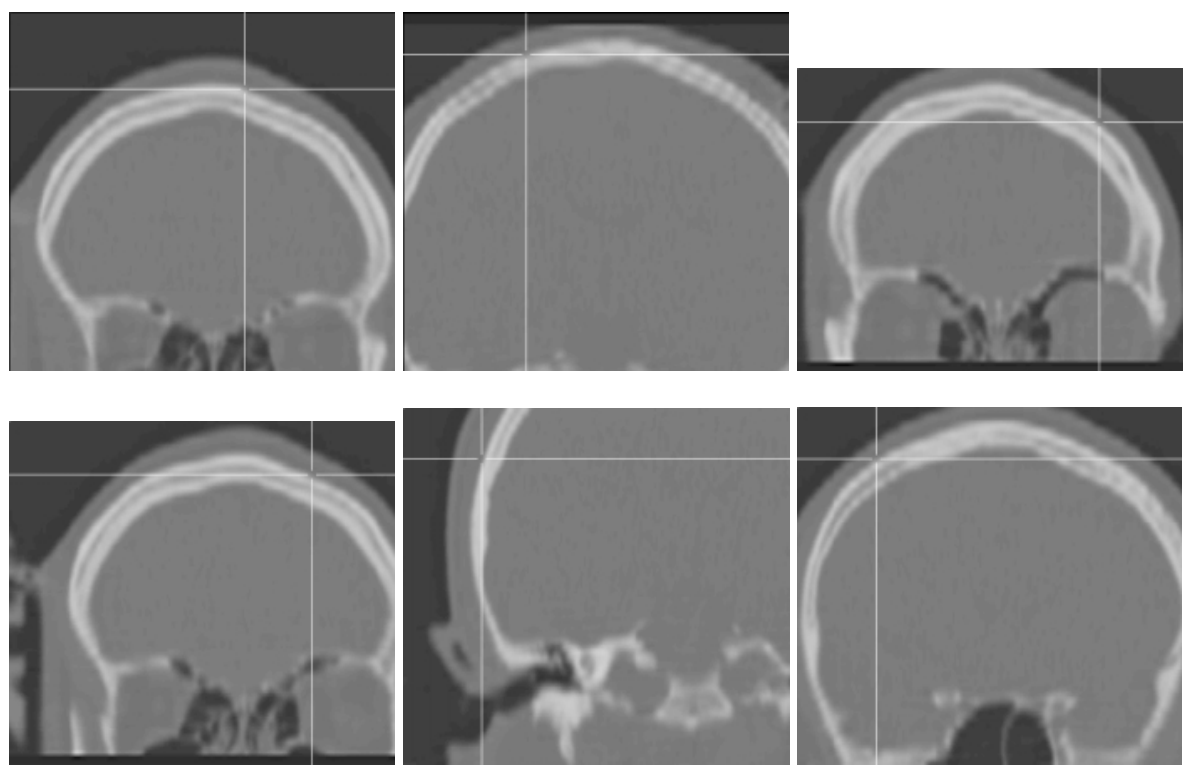

Fig. 5. Sample image slices from the patient CT scan with AcouStick recorded points overlaid as white crosses. The transformation between the AcouStick data and the segmented CT skull surface was determined using surface matching. The slices shown indicate the position of a representative group of six out of 30 points collected from this patient.

was extracted from the patient's CT image volume. The surface residual error is $0.17 \mathrm{~mm}$. Sample CT slices showing six of these surface points superimposed using this surface-based registration are shown in Fig. 5.

\section{Discussion}

We have presented a location device, the AcouStick, for image-to-physical registration in image-guided surgery. The device is based on a tracked A-mode ultrasound transducer. It can be considered to be a point localizer, with a variable length tip, capable of non-invasive penetration of soft tissue and fluids to locate echogenic structures such as bone. Our work builds on the work of Bass, Galloway \& Maurer [1], and is related to preliminary work reported by Schmerber et al. [9]. Neither of these earlier papers describe an appropriate calibration technique nor do they contain a detailed validation.

We have described the construction, data analysis, calibration, and initial work on validation of the system. The intrinsic accuracy of the system was measured using an array of steel balls. The free hand measurements, which suggest an accuracy of $0.5 \mathrm{~mm}$, will include latency errors in sensing the probe 
position and errors due to the finite width of the ultrasound beam $(-6 \mathrm{~dB}$ beam width is about $0.4 \mathrm{~mm}$ at the focal point). The fixed orientation measurements, which suggest an accuracy of $0.15 \mathrm{~mm}$, might underestimate systematic errors in the direction of the ultrasound beam. In practice the system will be used to measure points on surfaces with a radius of curvature much greater than $0.4 \mathrm{~mm}$, but latency errors and systematic errors will be important. Hence we regard 0.15 and $0.5 \mathrm{~mm}$ as the lower and upper bounds of the intrinsic accuracy of the system, giving it accuracy comparable to existing contact based pointers.

A phantom test on a skull, with gelatin covering to mimic scalp tissue, showed that the AcouStick could recover image-to-physical registration with a TRE of between 1.0 and $1.7 \mathrm{~mm}$. Location of skull surface points on MR scans of three volunteers wearing a lockable acrylic dental stent (LADS) for registration produced results within 1 or 2 voxels $(1$ to $2 \mathrm{~mm}$ ) for two volunteers. For the third volunteer there was a systematic shift of about 3 voxels. Independent experiments [3] yielded a mean TRE for relocation of the LADS for the three volunteers of $0.6,0.7$, and $1.1 \mathrm{~mm}$, respectively. Geometrical distortion, scaling errors, fat shift, etc., could be as high as $2 \mathrm{~mm}$ in these MR scans. These experiments therefore indicate an upper limit of the in vivo TRE of about $3 \mathrm{~mm}$ and are consistent with a TRE of between 1 and $2 \mathrm{~mm}$. Finally, the data was used to register a CT scan of a volunteer to physical space. This yielded a residual surface registration error of $0.17 \mathrm{~mm}$.

Preliminary results obtained from three volunteers and a patient indicate that the AcouStick has the potential to provide accurate image-to-physical registration of the head without the use of any markers or frames. Calibration currently takes about 20 minutes, and data collection in the operating theater about 15 minutes. The AcouStick may also be usable for non-cranial image-guided surgery applications.

\section{Acknowledgment}

C. R. Maurer, Jr. was a Research Fellow at King's College London when this work was performed. He was supported by the EASI project "European Applications for Surgical Interventions" HC1012 of the EU "4th Framework Telematics Applications for Health" program. We are grateful to Philips Medical Systems (Best, The Netherlands) for supporting R. Gaston and for supplying the equipment used in this project. P. J. Edwards was supported by the EPSRC GR/L62221. We thank G. Penney for his help and Dr. F. Gerritsen (Philips Medical Systems) for his advice, encouragement, and support. 


\section{References}

1. WA Bass, RL Galloway Jr, CR Maurer Jr. Surface-based registration of physical space with CT images using A-mode ultrasound localization of the skull. Medical Imaging 1998: Image Display, Proc. SPIE 3335: 228-238, 1998. 961

2. PJ Besl, ND McKay. A method for registration of 3-D shapes. IEEE Trans. Pattern Anal. Mach. Intell., 14: 239-256, 1992. 958

3. PJ Edwards, AP King, CR Maurer Jr, DA de Cunha, DJ Hawkes, DLG Hill, RP Gaston, MR Fenlon, S Chandra, AJ Strong, CL Chandler, A Richards, MJ Gleeson. A system for microscope-assisted guided interventions (MAGI). MICCAI '99 (appears elsewhere in these proceedings). 962

4. A Gueziec, R Hummel. Exploiting triangulated surface extraction using tetrahedral decomposition. IEEE Trans. Visualization Comput. Graph., 1: 328-342, 1995. 958

5. CR Maurer Jr, GB Aboutanos, BM Dawant, RJ Maciunas, JM Fitzpatrick. Registration of 3-D images using weighted geometrical features. IEEE Trans. Med. Imaging, 15: 836-849, 1996. 958

6. CR Maurer Jr, JM Fitzpatrick, MY Wang, RL Galloway Jr, RJ Maciunas, GS Allen. Registration of head volume images using implantable fiducial markers. IEEE Trans. Med. Imaging, 16: 447-462, 1997. 958, 959

7. CR Maurer Jr, RJ Maciunas, JM Fitzpatrick. Registration of head CT images to physical space using a weighted combination of points and surfaces. IEEE Trans. Med. Imaging, 17: 753-761, 1998. 958

8. W. W. McDicken. Diagnostic Ultrasonics: Principles and Use of Instruments. John Wiley \& Sons, Inc., New York, 1976. 954

9. S Schmerber, B Chen, S Lavallee, JP Chirossel, P Cinquin, A Poyet, M Coulomb, E Reyt. Markerless hybrid registration method for computer assisted endoscopic ENT surgery. In: Computer Assisted Radiology and Surgery 1997. HU Lemke, MW Vannier, K Inamura, eds. Springer-Verlag, Berlin, 1997, pp. 799-806. 961

10. R Sibson. Studies in the robustness of multidimensional scaling: Perturbational analysis of classical scaling. J. R. Statist. Soc. B, 41: 217-229, 1979. 958 